Silvia Meschi, Giulia Matusali, Francesca Colavita, Daniele Lapa, Licia Bordi, Vincenzo Puro, Bruno D. Leoni, Claudio Galli, Maria Rosaria Capobianchi* and Concetta Castilletti, on behalf of INMI Covid-19 laboratory and investigation team

\title{
Predicting the protective humoral response to a SARS-CoV-2 mRNA vaccine
}

https://doi.org/10.1515/cclm-2021-0700

Received June 15, 2021; accepted August 23, 2021;

published online September 8, 2021

\author{
Abstract \\ Objectives: Simple and standardized methods to establish \\ correlates to vaccine-elicited SARS-CoV-2 protection are \\ needed.
}

Methods: An observational study on antibody response to a mRNA vaccine (Comirnaty) was performed on health care workers (V, n=120). Recovered COVID-19 patients (N, n=94) were used for comparison. Antibody response was evaluated by a quantitative anti-receptor binding domain IgG (anti-RBD) commercial assay and by virus microneutralization test (MNT), in order to establish a threshold of anti-RBD binding antibody units (BAU) able to predict a robust ( $\geq 1: 80$ ) MNT titer.

Results: Significant correlation between BAU and MNT titers was found in both $\mathrm{V}$ and $\mathrm{N}$, being stronger in $\mathrm{V}$ ( $\mathrm{rs}=0.91$ and 0.57 respectively, $\mathrm{p}<0.001$ ); a higher incremental trend starting from MNT titer 1:80 was observed in the $\mathrm{V}$ group. The $99 \%$ probability of high MNT titer $(\geq 1: 80)$ was reached at 1,814 and 3,564 $\mathrm{BAU} / \mathrm{mL}$, and the area under the receiver operating characteristic (ROC) curve was 0.99 (CI: 0.99-1.00) and 0.78 (CI: 0.67-0.86) in V and N, respectively.

Silvia Meschi and Giulia Matusali contributed equally to the study.

The collaborative group is detailed at the end.

*Corresponding author: Dr. Maria Rosaria Capobianchi, Laboratory of Virology, National Institute for Infectious Diseases “L. Spallanzani”, Via Portuense 292, 00149 Rome, Italy,

E-mail: maria.capobianchi@inmi.it. https://orcid.org/0000-00020270-1711

Silvia Meschi, Giulia Matusali, Francesca Colavita, Daniele Lapa, Licia Bordi, Vincenzo Puro and Concetta Castilletti, National Institute for Infectious Diseases “Lazzaro Spallanzani” IRCCS, Rome, Italy

Bruno D. Leoni, Validation \& Verification, Core Laboratory, Abbott, Italy

Claudio Galli, Global Medical Affairs, Core Laboratory, Abbott, Italy
Conclusions: A threshold of 2,000 BAU/mL is highly predictive of strong MNT response in vaccinated individuals and may represent a good surrogate marker of protective response. It remains to be established whether the present results can be extended to BAU titers obtained with other assays.

Keywords: antibody response; protective immunity; SARS-CoV-2; vaccine; viral neutralization.

\section{Introduction}

SARS-CoV-2 pandemic has led to more than 210 million cases worldwide and more than four million deaths [1]. The former number is underestimated by an insufficient testing coverage in several countries and by at least $30 \%$ of cases of SARS-CoV-2 infection being asymptomatic [2]. Therefore, the real burden of the infection is not known, and this affects the implementation of public health strategies to curb the spread and limit the consequences. Containment measures based on physical barriers, social distancing and various degrees of 'lockdown' are effective [3] but cannot be maintained for long periods, out of need to provide economic and social relief. The best way to halt or reduce those measures is to cover a high share of the population by vaccination. Several COVID-19 vaccines, by different techniques, have been made available in a record time, thanks to the previous experience with similar viruses $[4,5]$. Vaccines based on mRNA have been available since December last year and mass vaccination campaigns started then.

Serological assays for virus-specific antibody response are commonly employed to establish the immunological response to vaccines and to establish correlates of protection [6]. While official policy recommendations do not include antibody testing as part of the vaccination programs, serological surveillance has been included in several pilot settings, and many studies have already established the extent and magnitude of antibody response following the administration of mRNA vaccines [7-9]. However, current serological assays detect binding antibodies, while the efficacy of the humoral immune response 
to SARS-CoV-2 is linked to neutralizing antibodies, that are not routinely detected [10]. We aimed to establish the usefulness of a recently developed immunoassays for the quantitation of IgG antibody response to SARS-CoV-2 receptor binding domain (anti-RBD) in the prediction of a strong neutralizing antibody response to an mRNA-based vaccine.

\section{Materials and methods}

\section{Study population}

Two groups have been included in our study. The first one included 120 health care workers (HCW) from the National Institute for Infectious Diseases "L. Spallanzani" who have been administered two doses of the BNT162b2 mRNA COVID-19 vaccine (Comirnaty) three weeks apart from December to February 2021 (V). Those subjects have been tested for binding and neutralizing antibody response at the time of first dose (T0), just before the second vaccine dose (T1), and two weeks after the second vaccine dose (T2). Out of those, 90 were naïve to SARS-CoV-2 (NA), while 30 experienced previous SARS-CoV-2 infection (PI) with mild or no symptoms. The second group, selected for comparison, included 94 patients who recovered from natural SARS-CoV-2 infection (N), whose serum samples were obtained between 67 and 295 days after the onset of symptoms.

\section{SARS-CoV-2 antibody testing}

The levels of binding IgG to SARS-CoV-2 RBD have been determined by an automated chemiluminescent immunoassay (ARCHITECT SARSCoV-2 IgG II Quantitative, Abbott Laboratories, Wiesbaden, Germany) described elsewhere [7]. The results are expressed in arbitrary units per milliliter $(\mathrm{AU} / \mathrm{mL})$ within a linear range spanning from 50 (positivity threshold) to 40,000, expanded to 80,000 with an automated dilution. Assay linearity throughout this range has been confirmed by an independent evaluation [7]. After the release of a WHO standard preparation for SARS-CoV-2 binding antibodies [11], a conversion factor from Abbott AU became available $(1 \mathrm{BAU} / \mathrm{mL}=0.142 \times \mathrm{AU} / \mathrm{mL})$ and the results obtained in this study have been expressed in BAU/mL. SARS-CoV-2 IgG antibodies directed to Nucleocapsid antigens (anti-N) have been determined by an automated chemiluminescent immunoassay (ARCHITECT SARS-CoV-2 IgG), as previously described [12]. Finally, a SARS-CoV-2 microneutralization assay (MNT) was performed on all samples by the method routinely employed in our laboratory [13]. The neutralization titer was assigned as the highest serum dilution inhibiting at least $90 \%$ of the cytopathic effect (IC90), with a positivity threshold set at 1:10. For the purpose of this study 1:80 was the minimum MNT titer to consider a serum sample as having a strong neutralizing capacity.

\section{Statistical analysis}

Results were presented as mean \pm standard deviation (SD) for continuous variables, medians with interquartile ranges (IQR) when non-normally distributed, and as a percent for categorical variables.
Clopper Pearson or Wald 95\% Confidence Intervals were properly indicated. Differences between average values for continuous or median for non-continuous variables were compared using ANOVA or the Kruskal-Wallis test/Wilcoxon signed-rank test or Steel-DwassCritchlow-Fligner all pairs comparisons. Statistical power was calculated >0.8. Dataset was built in Microsoft Excel 2019 (Microsoft TM), and statistical analysis was performed with Analyze-it 4.92.4, GraphPad Prism (version 9.0.2) and R software (version 3.6.0) with the tidyverse and ggplot2 packages.

The distribution of anti-RBD levels was evaluated in each group and by age ( $<$ or $\geq 50$ years) and gender; for $\mathrm{V}$ group, subjects with (previous positive RNA test or anti-RBD positive at T0) or without a previous exposure to SARS-CoV-2 have been considered. The comparison between anti-RBD and neutralizing antibodies has been studied using different statistical methods: a) positive and negative concordance by $2 \times 2$ contingency tables; b) distribution of anti-RBD levels according to neutralization titers by non-parametric analysis. Distributions data were represented in box-and-whiskers format, where the band within the box represents the median, box lower and upper border, 25th and 75th percentiles of the distribution, respectively. The lower and upper fences were calculated as per Tukey formula (Q1 - 1.5 × IQR; Q3 + 1.5IQR). Outliers were not eliminated and considered in calculations; c) correlation by Spearman method and receiver operating characteristic (ROC) curve analysis; d) logistic regression; involved descriptors interactions were also evaluated by average interaction plot (Supplementary Figure 1). Patients without a history of SARS-CoV-2 infection or vaccination were excluded from calculations. Moreover, for vaccinated subjects only samples collected after at least one dose of vaccine were included in the analyses. A Generalized Linear Model (GLM) per binomial repartition was built. Akaike information criterion (AIC) stepwise was applied for descriptor significance comparison, and the derived models were used for building the probability curves. The Goodness of Fit was evaluated with Hosmer and Lameshow test (Supplementary Table 1). A level of significance (p) $<0.05$ for two-sided distributions was applied for all tests.

\section{Ethics}

The INMI Ethical Committee approved the study "Exploiting immunophatogenic mechanisms for establishing predictive markers, diagnostic tools and medical countermeasures in COVID-19" (issue n. 14/ 2005 and subsequent amendment n.3/2019) and the study "Monitoring immune response to BNT162b2 mRNA COVID-19 vaccine in Health Care Workers" (issue n. 297/2021). All participants signed informed consent.

\section{Results}

\section{Population characteristics}

The V group included 120 HCW (80 females and 40 males) with a mean age of $47.1 \pm 11.8$ years (median: 48; range: $23-$ 71; no significant difference between genders). Out of those, 90 (59 females and 31 males) were naïve to SARS-CoV-2 (NA), and 30 (21 females and 9 males) had 
been previously infected (PI). The $\mathrm{N}$ group included 94 adult subjects ( 27 females and 67 males) with a mean age of $56.0 \pm 12.9$ years (median: 55.5 ; range: $20-86$; no significant difference between genders).

All patients in the $\mathrm{N}$ group were positive for anti-RBD with median levels of $525 \mathrm{BAU} / \mathrm{mL}$ (range: 15-7,566 $\mathrm{BAU} / \mathrm{mL}$ ), slightly higher on samples taken less than 90 days from diagnosis (621.4 vs. 500.5; p=n.s.). Eightyseven subjects (92.6\%) presented anti-N antibodies, the seven anti-N negatives having been tested $\geq 89$ days after onset. MNT was positive on 86 samples $(91.5 \%)$ at a median titer of 1:40. Titers $\geq 1: 80$ were recorded in 42 samples $(44.7 \%)$. At T0, none of the vaccinated subjects in the NA subgroup was positive for anti-N antibodies, while 14/30 $(46.7 \%)$ in the PI subgroup were positive. None of the former seroconverted for anti-N during the study period.

\section{Anti-RBD levels comparison in vaccinated or naturally infected people}

Anti-RBD levels after natural infection or vaccination are shown in Figure 1. Non infected-non vaccinated individuals were included as negative control group. $\mathrm{N}$ group showed high antibody levels (median: 525.1, IQR: 236.1-1,036).
In previously infected subjects (PI), a single dose (T1) of mRNA vaccine elicited a significant, 77-fold boost from the antibody titer measured at T0 (T0 median: 37, IQR: 22.9-102.5 vs. T1 median: 2,850, IQR: 1,535-4,047; $\mathrm{p}<0.001)$. In the NA group, anti-RBD reached a median level of 127.5 (IQR: 68.8-279) after a single dose (T1), with a further 19-fold increase after two doses (T2 median: 2,422 IQR: 1,895-3,621; $\mathrm{p}<0.001)$. Antibody levels were comparable in PI at T1 and NA at T2 subjects $(\mathrm{p}=0.858)$. Similar anti-RBD levels were observed in PI at T0 and NA at T1 subjects $(\mathrm{p}=0.06)$ (Figure 1$)$; the values at T0 were comparable to those observed in a group with mild infection (data not shown).

Anti-RBD levels in individuals who received at least one dose of vaccine $(\mathrm{V})$ and in the $\mathrm{N}$ group were investigated in relation to the titers of SARS-CoV-2 neutralizing antibodies. A significant correlation between anti-RBD IgG levels and the MNT titer was found in both $\mathrm{V}$ and $\mathrm{N}$ groups ( $\mathrm{rs}=0.91$ and 0.57 respectively, $\mathrm{p}<0.001$ ). A side by side non-parametrical distribution analysis (Figure 2) showed a positive correlation between anti-RBD levels and the neutralization titers in both groups. While no significant differences were recorded between $\mathrm{V}$ and $\mathrm{N}$ for neutralization titers comprised between $1: 10$ and $1: 40$, a highly significant $(p<0.001)$ increase was found for the $V$ group when comparing the 1:40 to 1:80 titers.

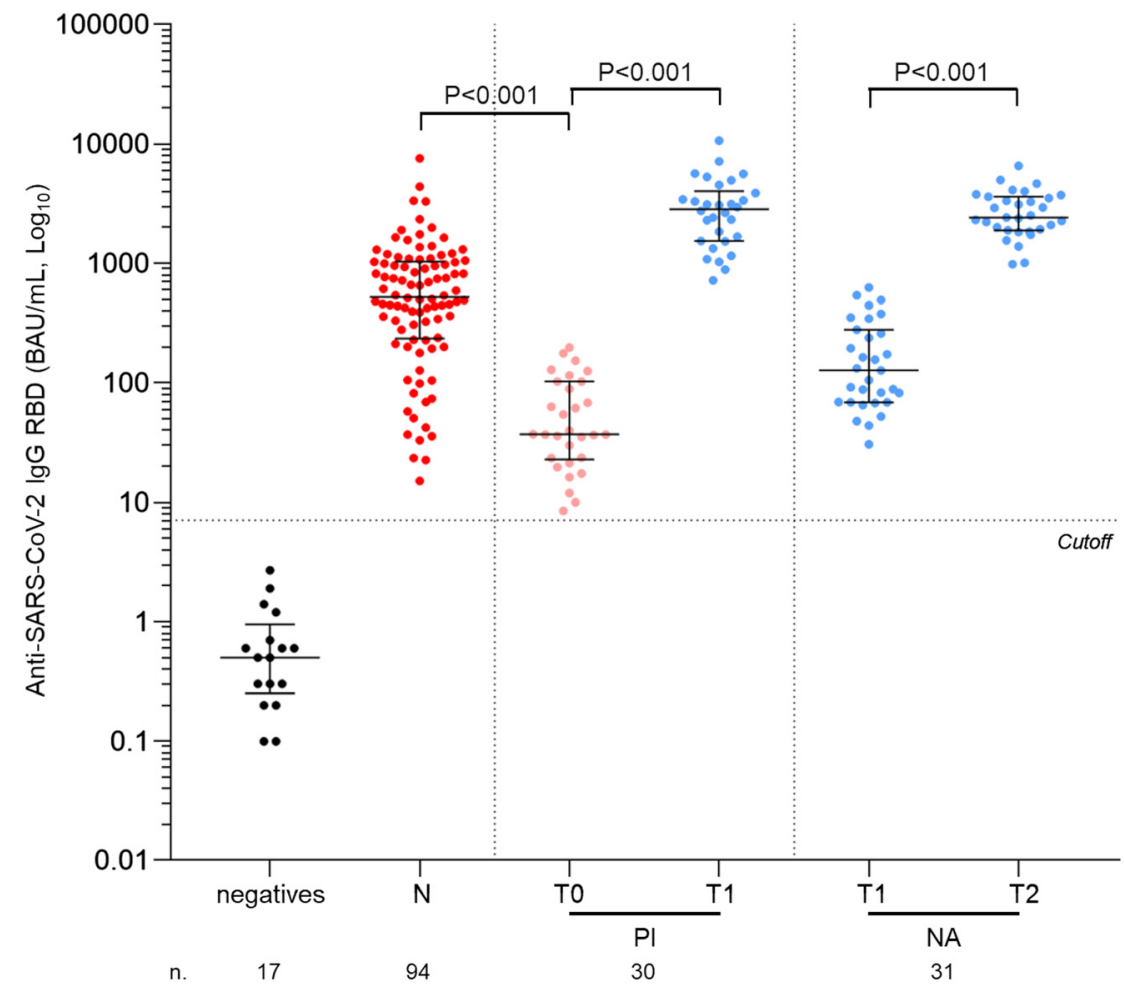

Figure 1: Distribution anti-SARS-CoV-2 IgG receptor binding domain (RBD) levels $\left(\log _{10} B A U / m L\right)$ in natural infection $(N)$ and after vaccination.

Horizontal lines in depicted jittered populations represent the median, whiskers the 25th and 75th percentile. Significant $p$-values are indicated over the brackets. The horizontal dotted line indicates the positivity cutoff value (7.1 BAU/mL). Vertical dotted lines divide negative and naturally infected from vaccinated individuals, and among the latter those who were naïve to the infection (NA) from those with a previous infection (PI). Only individuals with paired results available are shown in the NA and PI groups. $\mathrm{TO}=$ before the first dose of Comirnaty vaccine; $\mathrm{T} 1=$ three weeks after the first dose of Comirnaty vaccine; $\mathrm{T} 2=$ two weeks after the second dose of Comirnaty vaccine. At the baseline (TO) health care workers that experienced previous SARS-CoV-2 infection with mild or no symptoms were represented by pink dots. $\mathrm{n}=$ number of patients tested. Seventeen samples from non infected-non vaccinated subjects naïve to SARS-CoV-2 infection have been included in the figure. 


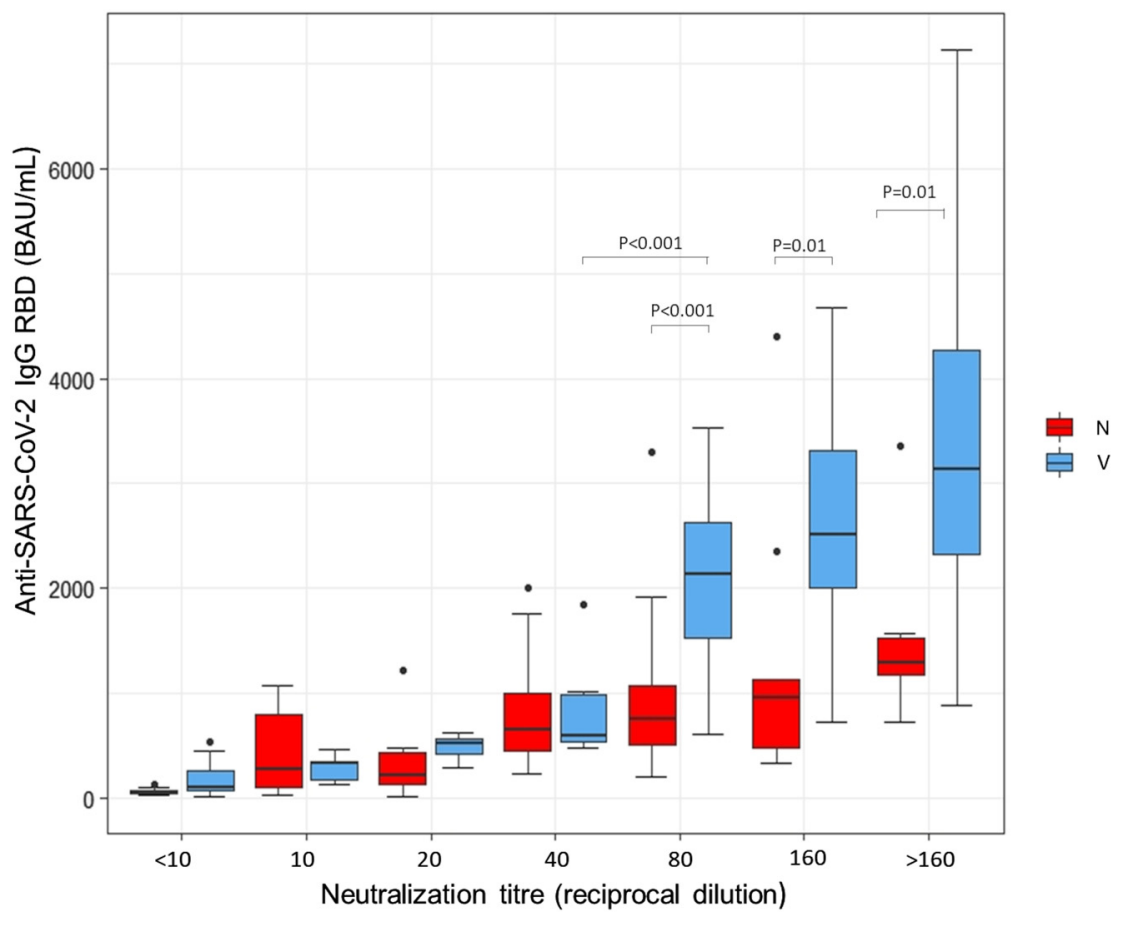

Figure 2: Box-and-whiskers plot of antiSARS-CoV-2 IgG receptor binding domain (RBD) levels $(B A U / m L)$ at different microneutralization (MNT) IC90 dilution titers in naturally infected $(\mathrm{N})$ and vaccinated subjects after receiving at least one dose (V). Box plots indicate the median and interquartile range (IQR); the whiskers represent 1.5 times the IQR. Significant p-values are indicated over the brackets.
Consistent with these findings, significant difference was observed when comparing antibody levels between the $\mathrm{N}$ and V groups starting from MNT 1:80 (1:80: p<0.001; 1:160 and >1:160: $p<0.001$ respectively). The antibody level dispersion at 1:80 or higher MNT titer suggested a neat separation of population distribution. Considering the neutralization values $<1: 80$ in both the $\mathrm{V}$ and $\mathrm{N}$ populations, any point was distributed below a 2,000 BAU/mL threshold while at $\geq 1: 80$ neutralization titers, the anti-RBD levels were distributed through the entire range even if in an unbalanced fashion as the $\mathrm{V}$ group showed higher antibody levels (see Supplementary Figure 2).

\section{Anti-RBD levels by age and gender}

Subjects aged $\geq 50$ years showed higher median values ( 427 vs. $58 \mathrm{BAU} / \mathrm{mL}$; $\mathrm{p}<0.001)$ in the $\mathrm{N}$ group and lower median values (524 vs. 1,208 $\mathrm{BAU} / \mathrm{mL} ; \mathrm{p}<0.001$ ) in the $\mathrm{V}$ group. Moreover median levels of anti-RBD antibodies were higher in males ( 412 vs. $63 \mathrm{BAU} / \mathrm{mL}$; $\mathrm{p}<0.001$ ) in the $\mathrm{N}$ group and in females ( 948 vs. $529 \mathrm{BAU} / \mathrm{mL}$; $\mathrm{p}<0.001$ ) in the $\mathrm{V}$ group (data not shown). However, differences by age and gender were not significant in both groups when anti-RBD median values were evaluated in comparison with neutralizing titers $\geq 1: 20$ or $\geq 1: 80$ (see Supplementary Table 2).

\section{Logistic regression and ROC curves}

The predictive value for MNT titers of anti-RBD levels has been calculated by logistic regression. Plots (Figure 3) show the probability for the $\mathrm{V}$ and $\mathrm{N}$ groups at 1:80 neutralization titer, with a consistently faster flection in the $\mathrm{V}$ group. In the $\mathrm{N}$ group, the probability of having a neutralization effect in the absence of anti-RBD antibodies was statistically not null. The antibody thresholds, calculated by logistics curves, corresponding to $99 \%$ probability of achieving a neutralization titer $\geq 1: 80$ were $1,814 \mathrm{BAU} / \mathrm{mL}$ (95\% CI: 939-4,393) for the $\mathrm{V}$ group and 3,564 BAU/mL (95\% CI: 1,970-9,471) for the $\mathrm{N}$ group (Table 1). Thresholds for the other neutralization titers are also reported (Table 1, see Supplementary Table 1).

ROC curves were developed separately for the two groups to assess sensitivity and specificity (Figure 4). Areas under the curves (AUC) were 0.78 (CI: 0.67-0.86) for the $\mathrm{N}$ group and 0.99 (CI: 0.99-1.00) for the $\mathrm{V}$ group and the cutoff by the Youden index was 480 and $610 \mathrm{BAU} / \mathrm{mL}$, respectively. Sensitivity thresholds at $99 \%$ specificity were also evaluated. For the $\mathrm{N}$ group, criterion values $>1,997$ BAU/mL showed a very low sensitivity (12\%), while for the $\mathrm{V}$ group a criterion $>1,836 \mathrm{BAU} / \mathrm{mL}$ showed a good sensitivity (79\%) (Figure 4). 


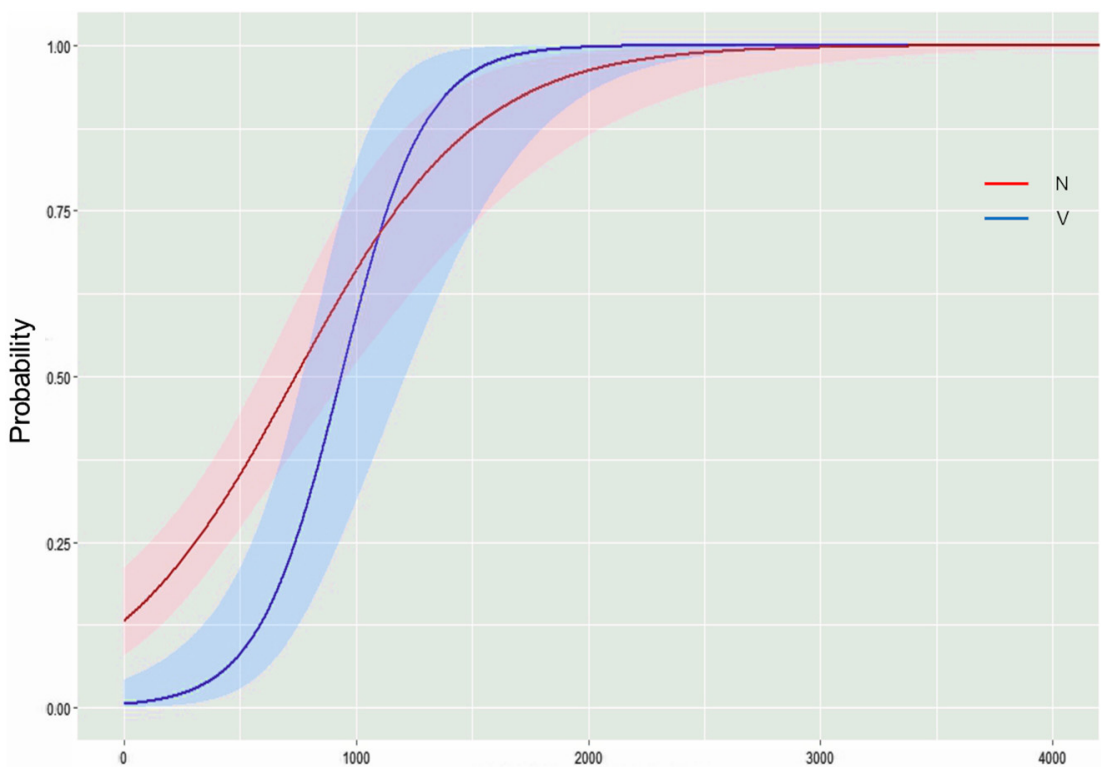

Anti-SARS-CoV-2 IgG RBD (BAU/mL)
Figure 3: Probability curves with $95 \%$ confidence intervals from a multivariate logistic regression model.

The two curves fit anti-SARS-CoV-2 IgG receptor binding domain (RBD) levels (BAU/ $\mathrm{mL}$ ) to the microneutralization (MNT) IC90 dilution response $(0=\langle 1: 80 ; 1=\geq 1: 80)$ in naturally infected $(\mathrm{N})$ vs. vaccinated (V) patients. The following logit equations describe the plotted model: Natural infection $(\mathrm{N})=-1.425+0.0017$ Anti-RBD (BAU/mL); Vaccination $(V)=-4.875+0.0052$ Anti-RBD (BAU $/ \mathrm{mL})$. The different colors are related to naturally infected (N) or vaccinated (V) individuals. Shadows represents $95 \%$ confidence interval (Cl).
Table 1: Levels of anti-SARS-CoV-2 IgG RBD (BAU/mL) calculated by multivariate logistic regression models (see Supplementary Table 1) corresponding to a $99 \%$ probability of being at or above different microneutralization IC90 dilution titers; $95 \%$ upper and lower confidence intervals are indicated in brackets.

\begin{tabular}{lrr}
\hline \multicolumn{3}{l}{ Anti-SARS-CoV-2 IgG (RBD) BAU/mL at 99\% probability } \\
\hline MNT(IC90) dilution & $\mathbf{N}[95 \% \mathrm{CI}]$ & $\mathbf{V}[\mathbf{9 5 \%} \mathrm{CI}]$ \\
\hline $1: 20$ & $1,109[745-4,029]$ & $1,197[822-2,248]$ \\
$1: 40$ & $1,507[868-3,279]$ & $833[562-1,014]$ \\
$1: 80$ & $3,564[1,970-9,471]$ & $1,814[939-4,393]$ \\
$1: 160$ & $6,285[3,272-21,358]$ & $4,987[3,177-8,735]$ \\
\hline
\end{tabular}

RBD, receptor binding domain; BAU, banding antibody unit; IC90, inhibitory concentration at $90 \%$; $\mathrm{Cl}$, confidence interval; $\mathrm{N}$, naturally infected; $V$, vaccinated.

\section{Discussion}

Vaccination efficacy may be evaluated on different outcome measures, i.e., the ability to prevent disease or infection, or even preventing the spread of the infection in a given population [4]. The available data on SARS-CoV-2 vaccines based on mRNA indicate that active immunization by any of those shall guarantee a high level of protection towards COVID-19 and its consequences [14, 15]. However, efficacy levels assessed during clinical trials may not reflect the effectiveness of the same vaccines in real-life scenarios [4]. Moreover, the emergence of the so-called 'variants of concern' (VOC), i.e., variant strains that carry mutations potentially able to reduce the neutralizing efficacy after either natural infection or vaccination has further increased the risk of a diminished efficacy [16].

To date, there is no standardized and verified definition for a specific correlate of protection from COVID-19 or SARS-CoV-2 infection, nevertheless, vaccine protective efficacy correlates with neutralizing antibody titers achieved upon vaccination $[15,17]$.

Commercially available assays detect binding antibodies while protective immunity is more likely linked to the neutralizing antibody response, and the most potent neutralizing antibodies are those against SARS-CoV-2 RBD [18].

In the present study, using an automated assay we tried to assess a robust threshold for anti-RBD levels that will predict a strong neutralizing response [17], both in naturally immune and in vaccinated individuals [7].

As we show in the present study, vaccines elicit a rapid and strong antibody response to their immunogenic components $[7,8]$ that is enhanced when vaccines are administered to subjects who experienced a previous infection $[7,9,19-21]$.

Our data confirm and expand on previous findings [19, 22]: both naturally immune and vaccinated individuals showed a high degree of concordance between anti-RBD antibodies and MNT titers, and the association is tighter among vaccinated, with a ROC AUC close to 1 . The higher concordance in vaccinated individuals may be explained by timing; in fact, in the present study testing has been carried out at specific timeframes after vaccination, while samples from recovered patients have been drawn at 

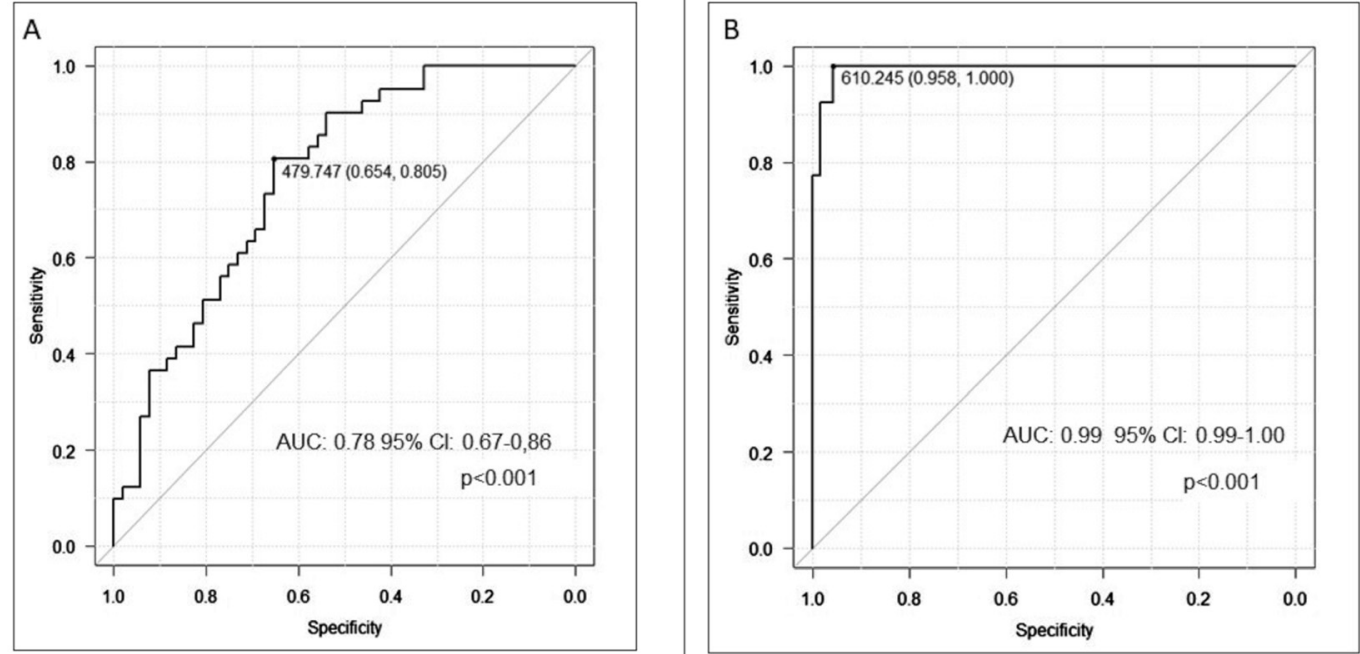

\begin{tabular}{|c|c|c|}
\hline Specificity & Sensitivity & Criterion \\
\hline 0.99 & 0.12 & $>1,997$ \\
\hline
\end{tabular}

\begin{tabular}{|c|c|c|}
\hline Specificity & Sensitivity & Criterion \\
\hline 0.99 & 0.79 & $>1,836$ \\
\hline
\end{tabular}

Figure 4: Receiver-operating characteristics (ROC) curves for anti-SARS-CoV-2 IgG receptor binding domain (RBD) values (BAU/mL) at 1:80 microneutralization (MNT) IC90 dilution titer in naturally infected (A) and vaccinated (B) subjects.

The area under the curve (AUC) and the fitting $p$-value are included in the picture. The optimal cutoff point was defined as the minimum value of $(1 \text {-sensitivity })^{2}+(1 \text {-specificity })^{2}$. Sensitivity values at $99 \%$ specificity are indicated below; the related measured cutoff is referred to as criterion.

different time intervals from disease onset. Furthermore, it has been suggested that the immune response to vaccine differs from natural immunity. Greaney et al. [23] have demonstrated that the neutralizing activity of vaccineelicited antibodies is even more focused on the spike RBD than for infection-elicited antibodies, and Goel et al. [24] provided evidence of both serological response and immunological memory following mRNA vaccination that is distinct based on prior SARS-CoV2 exposure. In our study at MNT titers $\geq 1: 80$ subjects that received vaccination (V) had a higher level of anti-RBD comparing to the individuals with a previous SARS-CoV-2 natural infection and not vaccinated $(\mathrm{N})$. This can be due to the presence, in naturally infected individuals, of neutralizing antibodies other than anti-RBD (such as antibodies that recognize the $\mathrm{N}$-terminal domain of the $\mathrm{S}$ protein), while in vaccinated subjects a strong anti-RBD response is present [25-28].

To our knowledge, this is the first study that provides evidence of a definite level of anti-RBD antibodies that predicts strong viral neutralization titers after SARS-CoV-2 vaccination.

Our study has some limitations. The number of subjects enrolled was relatively small, with an ensuing enlargement of the confidence intervals, and the demographic characteristics (age and gender) of the two groups considered were different. The latter, mentioned in other papers $[8,29]$ did not influence our results: while in general we found differences in anti-RBD levels according to age and between males and females, those differences were minimal and not significant when anti-RBD levels were compared to neutralizing titers (see Supplementary Table 2). Other factors to be considered are that results are specific for the anti-RBD assay and the neutralization method we have utilized. On the latter, we have employed a microneutralization assay that differs slightly from the 'classic' plaque reduction neutralization test (PRNT), as 96-well plates instead of 24-well plates are used [30], and allowed us to obtain an high throughput of samples analysis simultaneously. Moreover Bewley et al. showed an excellent correlation between PRNT and MNT [31]. However, the availability of reference standards for binding antibodies, such as the one recently provided by WHO, shall enable to harmonize results obtained by different methods [32]. The same is yet to come for viral neutralization methods [33], and this led us to select highly stringent conditions to define a strong neutralizing activity, i.e., a dilution titer of 1:80 at IC90. This may have caused an overestimation of the anti-RBD levels needed to attain a strong neutralization, but we preferred to privilege specificity in order to maximize the positive predictive value of 
those results. A second limitation is the different time of sampling after natural infection or vaccination. Indeed samples of individuals recovered from a previous SARS-CoV-2 natural infection and not vaccinated were obtained within a time span between 67 and 295 days after the onset of symptoms, whereas samples from vaccinated subject were collected after three (T1) or five (T2) weeks after the administration of first dose. A longer follow up of vaccinated individuals is in progress to evaluate possible differences in the time course of the antibody titers as well as in cellular response between vaccinated and individuals recovered from a natural infection. This will help to establish correlates of immunity, develop updated vaccines that will have efficacy towards the new SARS-CoV-2 variants or for populations that generally are not targeted by specific trials (pregnant women, children and immunosuppressed patients).

We believe that evaluating humoral immunity is relevant both clinically, for the subjects with a high likelihood of a weak response, and on a public health perspective. On the first point, Havlin et al. [34] have documented the absence of anti-SARS-CoV-2 antibodies, by three different assays, in patients undergoing lung transplantation (LTR) and vaccinated with the same vaccine employed in our study. Interestingly, LTR patients did show a good response after COVID-19, possibly due to a more complex and durable antigenic stimulation during natural infection. On the other side, Geisen et al. [35] did not find a lack of humoral response to mRNA vaccines in a cohort of patients with chronic inflammatory conditions and immunosuppressive therapy. Thus, the type and degree of pharmacological immunosuppression shall play a relevant role in shaping the immune response to vaccine in immunocompromised patients and the degree and amplitude of antibody response shall be verified in those categories.

Correlates to protection will help verify vaccine efficacy, establish when to administer vaccine doses beyond the current schedules, and determine the right time for a booster dose on the next 'flu season'. We are confident that our data may contribute on those purposes and look forward to other studies that may confirm this approach.

Research funding: The study was performed in the framework of the SARS-CoV-2 surveillance and response program implemented by the Lazio Region Health Authority. This study was supported by funds to the Istituto Nazionale per le Malattie Infettive (INMI) Lazzaro Spallanzani IRCCS, Rome (Italy), from Ministero della Salute (Programma CCM 2020; Ricerca Corrente - linea 1; COVID-2020-12371817); the European Commission -the European Virus Archive GLOBAL (grant no. 871029).
Author contributions: Conception and design of the study: S.M., G.M., V.P., M.R.C., C.C.; Laboratory investigation: S.M., G.M., F.C., D.L.; Data collection: S.M., G.M., V.P., C.C.; Data analysis: S.M., G.M., B.D.L., C.G.; Data interpretation: C.G., M.R.C., C.C.; Writing: S.M, G.M., C.G., M.R.C. with revisions and comments from all authors; Funding acquisition: M.R.C. INMI Covid-19 laboratory and investigation team contributed to the samples collection and storage and routine diagnostic. All authors have accepted responsibility for the entire content of this manuscript and approved its submission.

Competing interests: Dr. Claudio Galli and Dr. Bruno Daniele Leoni are currently employed by Abbott. Dr. Galli also owns Abbott stocks. All other authors have no competing interests.

Informed consent: Informed consent was obtained from all individuals included in this study.

Ethical approval: The INMI Ethical Committee approved the study "Exploiting immunophatogenic mechanisms for establishing predictive markers, diagnostic tools and medical countermeasures in COVID-19" (issue n. 14/2005 and subsequent amendment n.3/2019) and the study "Monitoring immune response to BNT162b2 mRNA COVID-19 vaccine in Health Care Workers" (issue n. 297/2021).

INMI Covid-19 laboratory and investigation team: Agrati Chiara, Abbate Isabella, Aiello Alessandra, Aleo Loredana, Amendola Alessandra, Alonzi Tonino, Arduini Nicolina, Berno Giulia, Bettini Aurora, Biancone Silvia, Biava Mirella, Bibbò Angela, Bonfiglio Giulia, Bordi Licia, Bordoni Veronica, Brega Carla, Capobianchi Maria Rosaria, Capri Andrea, Carletti Fabrizio, Casetti Rita, Castilletti Concetta, Ciafrone Lucia, Cimini Eleonora, Coen Sabrina, Colavita Francesca, Coppola Antonio, D’Alessandro Gaetano, Di Caro Antonino, Di Filippo Stefania, Fabeni Lavinia, Fard Said Najafi, Farrone Chiara, Federici Luigi, Felici Luisa, Ferraioli Valeria, Forbici Federica, Francalancia Massimo, Garbuglia Anna Rosa, Giombini Emanuela, Goletti Delia, Grassi Germana, Graziano Silvia, Gruber Cesare Ernesto Maria, Iazzetti Roberto, Lalle Eleonora, Lapa Daniele, Leone Barbara, Marafini Giovanni, Marchetti Federica, Mariotti Davide, Massimino Chiara, Matusali Giulia, Meschi Silvia, Messina Francesco, Minosse Claudia, Montaldo Claudia, Mucciante Mirco, Neri Stefania, Notari Stefania, Petrivelli Elisabetta, Petroni Fabrizio, Petruccioli Elisa, Pisciotta Marina, Pizzi Daniele, Prota Gianluca, Rotiroti Nicolina, Rozera Gabriella, Rueca Martina, Sabatini Rossella, Sacchi Alessandra, Santini Francesco, Santoro Anna Paola, Sarti Silvia, Sberna Giuseppe, Sciamanna Roberta, Scionti Rachele, Specchiarello Eliana, Tartaglia Eleonora, 
Valli Maria Beatrice, Vairo Francesco, Vanini Valentina, Vantaggio Valentina, Zambelli Emma.

\section{References}

1. Johns Hopkins University and Medicine. COVID-19 Dashboard by the Center for Systems Science and Engineering (CSSE) at Johns Hopkins University (JHU) [Internet]. Available from: https:// coronavirus.jhu.edu/map.html [Cited 21 Aug 2021].

2. Oran DP, Topol EJ. The proportion of SARS-CoV-2 infections that are asymptomatic. Ann Intern Med 2021;174:655-62.

3. Islam N, Sharp SJ, Chowell G, Shabnam S, Kawachi I, Lacey B, et al. Physical distancing interventions and incidence of coronavirus disease 2019: natural experiment in 149 countries. BMJ 2020:m2743. https://doi.org/10.1136/bmj.m2743.

4. Hodgson SH, Mansatta K, Mallett G, Harris V, Emary KRW, Pollard AJ. What defines an efficacious COVID-19 vaccine? A review of the challenges assessing the clinical efficacy of vaccines against SARS-CoV-2. Lancet Infect Dis 2021;21:e26-35.

5. Corey L, Mascola JR, Fauci AS, Collins FS. A strategic approach to COVID-19 vaccine R\&D. Science 2020;368:948-50.

6. Zimmermann P, Ritz N, Perrett KP, Messina NL, van der Klis FRM, Curtis N. Correlation of vaccine responses. Front Immunol 2021; 12. https://doi.org/10.3389/fimmu.2021.646677.

7. Narasimhan M, Mahimainathan L, Araj E, Clark AE, Markantonis J, Green A, et al. Clinical evaluation of the Abbott Alinity SARS-CoV2 spike-specific quantitative IgG and IgM assays among infected, recovered, and vaccinated groups. J Clin Microbiol 2021;59: e0038821.

8. Grupel D, Gazit S, Schreiber L, Nadler V, Wolf T, Lazar R, et al. Kinetics of SARS-CoV-2 anti-S IgG after BNT162b2 vaccination. Vaccine 2021;39:5337-40.

9. Ebinger JE, Fert-Bober J, Printsev I, Wu M, Sun N, Figueiredo JC, et al. Prior COVID-19 infection and antibody response to single versus double dose mRNA SARS-CoV-2 vaccination. medRxiv 2021 [Internet]. Available from: http://medrxiv.org/content/ early/2021/02/26/2021.02.23.21252230.abstract.

10. Fergie J, Srivastava A. Immunity to SARS-CoV-2: lessons learned. Front Immunol 2021;12. https://doi.org/10.3389/fimmu.2021. 654165

11. WHO. First WHO International Standard for anti-SARS-CoV-2 immunoglobulin; 2021 [Internet]. Available from: https://www. who.int/groups/expertcommittee-on-biological-standardization [Cited 28 Apr 2021].

12. Meschi S, Colavita F, Bordi L, Matusali G, Lapa D, Amendola A, et al. Performance evaluation of Abbott ARCHITECT SARS-CoV-2 IgG immunoassay in comparison with indirect immunofluorescence and virus microneutralization test. J Clin Virol 2020;129:104539.

13. Matusali G, Colavita F, Lapa D, Meschi S, Bordi L, Piselli P, et al. SARS-CoV-2 serum neutralization assay: a traditional tool for a brandnew virus. Viruses 2021:13. https://doi.org/10.3390/v13040655.

14. Goldberg Y, Mandel M, Woodbridge Y, Fluss R, Novikov I, Yaari R, et al. Protection of previous SARS-CoV-2 infection is similar to that of BNT162b2 vaccine protection: a three-month nationwide experience from Israel. medRxiv 2021 [Internet]. Available from: http://medrxiv.org/content/early/2021/04/24/2021.04.20. 21255670.abstract.
15. Earle KA, Ambrosino DM, Fiore-Gartland A, Goldblatt D, Gilbert $\mathrm{PB}$, Siber $\mathrm{GR}$, et al. Evidence for antibody as a protective correlate for COVID-19 vaccines. Vaccine 2021;39:4423-8.

16. Lumley SF, Rodger G, Constantinides B, Sanderson N, Chau KK, Street TL, et al. An observational cohort study on the incidence of SARS-CoV-2 infection and B.1.1.7 variant infection in healthcare workers by antibody and vaccination status medRxiv 2021 [Internet]. Available from: http://medrxiv.org/content/early/ 2021/03/12/2021.03.09.21253218.abstract.

17. Khoury DS, Cromer D, Reynaldi A, Schlub TE, Wheatley AK, Juno JA, et al. Neutralizing antibody levels are highly predictive of immune protection from symptomatic SARS-CoV-2 infection. Nat Med 2021;27:1205-11.

18. Andreano E, Nicastri E, Paciello I, Pileri P, Manganaro N, Piccini G, et al. Extremely potent human monoclonal antibodies from COVID-19 convalescent patients. Cell 2021;184:1821-35.e16.

19. Prendecki M, Clarke C, Brown J, Cox A, Gleeson S, Guckian M, et al. Effect of previous SARS-CoV-2 infection on humoral and $\mathrm{T}$-cell responses to single-dose BNT162b2 vaccine. Lancet (London, England) 2021;397:1178-81.

20. Krammer F, Srivastava K, Simon V. Robust spike antibody responses and increased reactogenicity in seropositive individuals after a single dose of SARS-CoV-2 mRNA vaccine medRxiv 2021 [Internet]. Available from: http://medrxiv.org/ content/early/2021/02/01/2021.01.29.21250653.abstract.

21. Manisty C, Otter AD, Treibel TA, McKnight Á, Altmann DM, Brooks $\mathrm{T}$, et al. Antibody response to first BNT162b2 dose in previously SARS-CoV-2-infected individuals. Lancet 2021;397:1057-8.

22. Cotugno N, Ruggiero A, Bonfante F, Petrara MR, Zicari S, Pascucci $G R$, et al. Virological and immunological features of SARS-CoV2-infected children who develop neutralizing antibodies. Cell Rep 2021;34:108852.

23. Greaney AJ, Loes AN, Gentles LE, Crawford KHD, Starr TN, Malone KD, et al. The SARS-CoV-2 mRNA-1273 vaccine elicits more RBD-focused neutralization, but with broader antibody binding within the RBD. bioRxiv 2021 [Internet]. Available from: http://biorxiv.org/content/early/2021/04/14/2021.04.14. 439844.abstract.

24. Goel RR, Apostolidis SA, Painter MM, Mathew D, Pattekar A, Kuthuru 0 , et al. Longitudinal analysis reveals distinct antibody and memory B cell responses in SARS-CoV2 naïve and recovered individuals following mRNA vaccination medRxiv 2021 [Internet]. Available from: http://medrxiv.org/content/early/2021/03/06/ 2021.03.03.21252872.abstract.

25. Chi X, Yan R, Zhang J, Zhang G, Zhang Y, Hao M, et al. A neutralizing human antibody binds to the $\mathrm{N}$-terminal domain of the Spike protein of SARS-CoV-2. Science 2020;369:650-5.

26. Yoshida S, Ono C, Hayashi H, Fukumoto S, Shiraishi S, Tomono K, et al. SARS-CoV-2-induced humoral immunity through $B$ cell epitope analysis in COVID-19 infected individuals. Sci Rep 2021;11:5934.

27. McCallum M, De Marco A, Lempp FA, Tortorici MA, Pinto D, Walls $\mathrm{AC}$, et al. $\mathrm{N}$-terminal domain antigenic mapping reveals a site of vulnerability for SARS-CoV-2. Cell 2021;184:2332-47.e16.

28. Suryadevara N, Shrihari S, Gilchuk P, VanBlargan LA, Binshtein E, Zost SJ, et al. Neutralizing and protective human monoclonal antibodies recognizing the $\mathrm{N}$-terminal domain of the SARS-CoV-2 spike protein. Cell 2021;184:2316-31.e15.

29. Pellini R, Venuti A, Pimpinelli F, Abril E, Blandino G, Campo F, et al. Early onset of SARS-COV-2 antibodies after first dose of BNT162b2: correlation with age, gender and BMI. Vaccines 2021;9:685. 
30. Focosi D, Maggi F, Mazzetti P, Pistello M. Viral infection neutralization tests: a focus on severe acute respiratory syndrome-coronavirus- 2 with implications for convalescent plasma therapy. Rev Med Virol 2021;31: e2170.

31. Bewley KR, Coombes NS, Gagnon L, McInroy L, Baker N, Shaik I, et al. Quantification of SARS-CoV-2 neutralizing antibody by wildtype plaque reduction neutralization, microneutralization and pseudotyped virus neutralization assays. Nat Protoc 2021;16: 3114-40.

32. Kristiansen PA, Page M, Bernasconi V, Mattiuzzo G, Dull P, Makar $\mathrm{K}$, et al. WHO International Standard for anti-SARS-CoV-2 immunoglobulin. Lancet 2021;397:1347-8.

33. Rogliani P, Chetta A, Cazzola M, Calzetta L. SARS-CoV-2 neutralizing antibodies: a network meta-analysis across vaccines. Vaccines 2021;9:227.
34. Havlin J, Svorcova M, Dvorackova E, Lastovicka J, Lischke R, Kalina T, et al. Immunogenicity of BNT162b2 mRNA COVID-19 vaccine and SARS-CoV-2 infection in lung transplant recipients. J Heart Lung Transplant 2021; 40:754-8.

35. Geisen UM, Berner DK, Tran F, Sümbül M, Vullriede L, Ciripoi M, et al. Immunogenicity and safety of anti-SARS-CoV-2 mRNA vaccines in patients with chronic inflammatory conditions and immunosuppressive therapy in a monocentric cohort. Ann Rheum Dis 2021. https://doi.org/10.1136/annrheumdis-2021220272.

Supplementary Material: The online version of this article offers supplementary material (https://doi.org/10.1515/cclm-2021-0700). 